\title{
Comorbidity of Narcolepsy and Obstructive Sleep Apnea: A Case Report
}

\author{
Hayeon Kim, MD ${ }^{1,2}$, Ji Hyun Song ${ }^{1}$, Tae Won Kim, MD ${ }^{1,2}$, Sung Min Kim, $\mathrm{MD}^{1,2}$, Yoo Hyun Um, MD, PhD ${ }^{1,2}$, \\ Ji Hye Oh, MD ${ }^{1,2}$, In Hee Cho, MD ${ }^{1,2}$, Seung Chul Hong, MD, PhD ${ }^{1,2}$ \\ 'Sleep Disorders Clinic, Department of Psychiatry, St. Vincent's Hospital, College of Medicine, The Catholic University of Korea, Suwon, Korea \\ ${ }^{2}$ Department of Psychiatry, St. Vincent's Hospital, College of Medicine, The Catholic University of Korea, Suwon, Korea
}

\author{
Received: November 27, 2018 \\ Revised: December 20, 2018 \\ Accepted: December 25, 2018 \\ Correspondence \\ Seung Chul Hong, MD, PhD \\ Department of Psychiatry, \\ St. Vincent's Hospital, College of Medicine, \\ The Catholic University of Korea, \\ 93 Jungbu-daero, Paldal-gu, \\ Suwon 16247, Korea \\ Tel $+82-31-249-7114$ \\ Fax +82-31-248-6758 \\ E-mail hscjohn@hotmail.com \\ ORCID \\ Hayeon Kim \\ https://orcid.org/0000-0002-7739-7152 \\ Ji Hyun Song \\ https://orcid.org/0000-0002-0128-2869 \\ Tae Won Kim \\ https://orcid.org/0000-0003-2689-202X \\ Sung Min Kim \\ https://orcid.org/0000-0002-3082-4014 \\ Yoo Hyun Um \\ https://orcid.org/0000-0002-3403-4140 \\ Ji Hye Oh \\ https://orcid.org/0000-0002-1935-4066 \\ In Hee Cho \\ https://orcid.org/0000-0003-0694-7249 \\ Seung-Chul Hong \\ https://orcid.org/0000-0003-0828-2906
}

Excessive daytime sleepiness (EDS) is the main symptom in narcoleptic patients. Obstructive sleep apnea (OSA) is known to be related to EDS. We describe three cases of narcolepsy that were misdiagnosed as other diseases or as OSA alone. Three patients with the onset of snoring and daytime sleepiness in adolescence were referred to our sleep center. Polysomnography showed severe sleep apnea. Their multiple sleep latency test (MSLT) met the diagnostic criteria of narcolepsy. Another patient who had EDS and snoring showed severe OSA and positive results on MSLT. However, longitudinal clinical symptoms of this patient differed from those of the three cases. EDS was ameliorated in all four patients following the treatment with psychostimulants and the application of Continuous positive airway pressure. Patients suffering from EDS may be co-diagnosed with narcolepsy and OSA. Clinicians should be aware of the possibility of false-positives of MSLT and the patient's longitudinal clinical course.

Sleep Med Res 2018;9(2):124-127

Key Words Narcolepsy, Obstructive sleep apnea, Polysomnography, Multiple sleep latency test, Continuous positive airway pressure. 


\section{CASE DESCRIPTION}

\section{Co-Diagnosis of Narcolepsy and OSA}

\section{Case 1-1}

On September 12, 2017, a 78-year-old male was referred to our sleeping center complaining of EDS, episodes of general weakness and a state being unable to move provoked by excessive emotions such as laughing or being upset. Since decades ago, he was witnessed snoring during the night. The patient had no difficulty falling asleep and he had a regular sleep schedule. According to the patient, he had his arm broken while practicing Chinese Kung Fu 30 years ago and reported no history of brain damage from the accident. The patient underwent the operation for his arm fracture and from then on, he started to experience symptoms of cataplexy episodes. When he laughed out loud, joked with his co-workers, or got frustrated when arguing with others, he fell down on his knees reporting sudden loss of muscle tone.

He regularly visited neurosurgery clinic and heard that the cause may be a transient ischemic vessel disease. Thus, he started to take aspirin for the prevention of heart attack. However, he performed various examinations including physical examination, MRI and MRA and EEG monitoring. No other medical problems were identified.

According to International Classification of Sleep Disorders (ICSD)-3, the prevalence of OSA increases with age. About $4 \%$ in middle-aged men (age 30-60 years) were reported have OSA [4]. The patient's snoring has worsened since 15 years ago and sometimes it seemed that he gasped and stopped breathing at night. At the same time, his EDS has started to get worsen and he got into a car accident crashing into the guardrail. Also his cataplexy episodes became more frequent since a year ago. The patient hasn't experienced any sleep paralysis or hypnagogic hallucination. He visited emergency room and neurosurgery clinic complaining of sudden loss of muscle power and was diagnosed with complex partial seizure. Even though he took prescribed antiepileptic medications, no symptom alleviation was made. Finally, he visited our clinic via neurosurgery clinic consultation.

He had neither depression nor any other psychiatric disorder. His BMI was $25.1 \mathrm{~kg} / \mathrm{m}^{2}$ which belongs to the obese group based on the clinical practice guidelines for overweight and obesity in Korea [5]. Also, his Epworth Sleepiness Scale (ESS) score was 14. Polysomnography (PSG) documented severe OSA with apnea-hypopnea index (AHI) of 44.7/h. Stage REM latency from sleep onset was 5.0 minutes. PSG also reported of 55.1\% of snore time during the night, and periodic limb movement in sleep (PLMS) reported 63.3/h. MSLT showed a mean sleep latency (MSL) of 1.2 min with four sleep onset REM periods (SOREMPs). The patient exhibited respiratory, apnea and hypopnea arousal. His limb movement arousal index was 16.4 out of total arousal index (61.0). The PSG data showed no cen- tral or mixed sleep apnea. The longest apnea period was 31.8 seconds, and the longest hypopnea period was 78.7 seconds. Mean oxygen saturation was $93.7 \%$ and the lowest saturation was $85.0 \%$. Saturation below $80 \%$ reported 11.5 minutes during total sleep time. Respiratory disturbance index (RDI) during REM sleep was 52.1 and 42.0 in NREM sleep. We did not carry out the genetic testing and measurement of cerebral spinal fluid (CSF) hypocretin concentration. Therefore, he was diagnosed with narcolepsy with cataplexy and OSA hypopnea, severe.

He started taking venlafaxine and modafinil first, and CPAP treatment was then applied after CPAP titration. His CPAP pressure was $7 \mathrm{cmH}_{2} \mathrm{O}$ and $\mathrm{AHI}$ decreased to $1.1 / \mathrm{h}$ following CPAP treatment. Clinical improvement after taking venlafaxine was shown. He did not have cataplexy events since taking venlafaxine. After two treatments, his ESS score was reduced to 6 , and reported overall ADL improvement.

\section{Case 1-2}

Another patient was a 28-year-old male who visited our sleep center on July 12, 2017. He didn't have a history of childhood obesity. Since his high school years, he experienced EDS and even collapsed while he tried to remain in the upright position to resist falling asleep. He fell asleep in classes. He was told by family members that he snores very loud and gasps during the night. He visited a local sleep center and was diagnosed with OSA. Although he had tonsillectomy, minimal improvement of EDS was made. He denied any sleep paralysis or hypnagogic hallucination.

The patient did not have any metabolic syndromes such as DM, HTN, and dyslipidemia. He also did not have depression or other psychiatric disorder. His BMI was $37.4 \mathrm{~kg} / \mathrm{m}^{2}$ and was obese by Korean obese standards [5]. His ESS score was 15. PSG reported AHI of 58.2/h, indicating severe OSA. Stage REM latency from sleep onset was 1.0 minute, and PLMS documented 17.8/h. MSLT showed a MSL of 1.5 min with four SOREMPs. His limb movement related arousal index was 4.0 out of total arousal index (58.0). The patient showed mixed and central sleep apnea patterns in addition to obstructive patterns. Central apnea index was $15.5 / \mathrm{h}$, and mixed apnea recorded $4.3 / \mathrm{h}$. The longest apnea period was 50.7 seconds, and the longest hyponea period was 59.5 seconds. Mean oxygen saturation was $95.3 \%$, and the lowest saturation was $76.0 \%$. Saturation below $90 \%$ was total 31.1 minutes and saturation below $80 \%$ was 0.3 minutes. The patient's RDI was 80.2 in REM sleep and 51.3 in NREM sleep. We did not measure the CSF hypocretin concentration to determine the cause and did not carry out any genetic testing.

As a result, he was also diagnosed with narcolepsy with cataplexy and OSA hypopnea, severe. We prescribed him modafinil $200 \mathrm{mg}$ daily. We also recommended him to take CPAP titration and to apply the device. However, the patient declined to take CPAP treatment due to financial difficulties. Weight re- 
duction was not the patient's main concern. He underwent neither bariatric surgery nor diet resection. Furthermore, no oral appliances were applied. He had not taken antidepressant for treatment of cataplexy, and the patient was lost to follow-up.

\section{Case 1-3}

On June 19, 2017 a 38-year-old male patient was referred to our sleep clinic. He suffered from EDS since middle school years but he had no specific cataplexy symptoms. He started to snore terribly loud since 15 years ago while he was sleeping and EDS got worsened. He was diagnosed with OSA by another clinician 6 years ago and was recommended to undergo tonsillectomy. Although he got the operation, the patient gained $25 \mathrm{~kg}$ of weight during the following two years because of irregular work and sleep pattern. He dozed while driving and got into a car accident. He was able to notice his EDS was a disabling condition.

According to the patient, he was not diagnosed with any of the psychiatric disorder previously, but he was under much stress from work since three years ago. He complained of depressive mood when he visited our sleep clinic. He was also anxious about his 9-year-old son being treated for depression. The patient's psychological test was performed and his Hamilton Depression Rating Scale (HAM-D) level was 12.

His BMI was $38.3 \mathrm{~kg} / \mathrm{m}^{2}$ and his ESS score was 18. PSG reported AHI of $85.1 / \mathrm{h}$, indicating severe OSA. Stage REM latency from sleep onset was 87 minutes, and he had no PLMS. MSLT showed a MSL of $1.4 \mathrm{~min}$ with three SOREMPs. The patient's arousal was only associated with respiratory arousals, apnea and hypopnea arousals. The PSG data showed central apnea (index $0.1 / \mathrm{h}$ ) and mixed sleep apnea (index 3.1/h). The longest apnea period was 77.7 seconds, and the longest hypopnea period was 92.2 seconds. Mean oxygen saturation was $85.9 \%$ and the lowest saturation was $62.0 \%$. Saturation below $90 \%$ reported 285.6 minutes, saturation below $80 \%$ reported 96 minutes, and saturation below $70 \%$ was 20.9 minutes during total sleep time. RDI during REM sleep was 69.4 and 90.6 in NREM sleep. Finally, he was diagnosed with narcolepsy without cataplexy and OSA hypopnea, severe. We prescribed him modafinil $200 \mathrm{mg}$ daily, but he complained of some side effects such as headache, myalgia, mood swings, agitation and skin rash. Therefore, we switched the medication from modafinil to methylphenidate. After taking the medication, his ESS score was 13 . He was also applied CPAP, with the pressure at $15 \mathrm{~cm}$ $\mathrm{H}_{2} \mathrm{O}$. After CPAP treatment, the patient reported the reduction of ESS score to 11.

\section{Case of False-Positive MSLT}

\section{Case 2-1}

On June 21, 2017 a 63-year-old male patient was referred to our sleep clinic. He was observed snoring since he was in his teens, but he kept a regular sleep pattern. Since a year ago, he easily began to fall asleep during daytime. His snoring also became louder and longer since six months ago, and the patient's EDS worsened in the middle of driving waiting for the signal.

He was previously diagnosed with diabetes mellitus, hypertension and benign prostatic hyperplasia, thus taking medications. His BMI was $35 \mathrm{~kg} / \mathrm{m}^{2}$ and his ESS score was 15 . PSG results showed a AHI of $97.8 / \mathrm{h}$, indicating severe OSA. Stage REM latency from sleep onset was 306 minutes, and he had no PLMS. MSLT showed a MSL of $3.5 \mathrm{~min}$ with two SOREMPs. He was diagnosed with OSA hypopnea, severe and also could be diagnosed with narcolepsy without cataplexy according to MSLT results.

Although he had positive MSLT results, his clinical process differed from case 1 patients. He did not experience EDS in his early years. Also, his EDS developed after his snoring became worse. The patient experienced sleep fragmentation and chronic insufficiency of sleep. This condition may have influenced the MSLT, and the positive result may be a false-positive result. CPAP is a suggested treatment for severe OSA patient. After the CPAP treatment, additional MSLT study may be helpful for differentiating narcolepsy.

\section{DISCUSSION}

These are case reports that demonstrate narcolepsy and OSA can be diagnosed simultaneously. Previous studies that examine the comorbidity of narcolepsy and OSA are very few. Only some case reviews of narcoleptic OSA patients have been reported [68]. In a study [9], many OSA patients complain about persistent sleepiness after CPAP treatment. In this case, various medical conditions and psychiatric conditions such as depression, and sleep disorders including narcolepsy and idiopathic hypersomnia should be examined preferentially. Also, Frauscher et al. [10] reported that $24 \%$ of patients with narcolepsy were diagnosed with sleep-related breathing disorders. The majority of them (21 of 24) had OSA, and ten people out of 24 OSA patients were reported to have a moderate to severe AHI. Narcoleptic patients were often obese in various studies $[11,12]$ and elevated BMI may be associated with the comorbidity of narcolepsy and OSA. Deficiency of hypocretin in narcoleptic patients may decrease basal metabolism and food intake, and may be a potential cause of obesity. Also, narcoleptic patients are known to suffer from a mild eating disorder, and usually lack of physical exercise [13].

In our first case, a 78-year-old patient's cataplexy symptoms were misdiagnosed as syncope, ischemic brain vessel disease, and complex partial seizure, delaying proper evaluation and management. One explanation for the patient's experience of EDS with cataplexy at age 48 is late onset narcolepsy [14]. The patient's cataplexy can be exhibited at any point following the onset of EDS. In case 1-2 and 1-3, narcoleptic patients without 
cataplexy who suffered from EDS since teens were mistaken as an OSA only patient. Although the two patients both had tonsillectomy, daytime sleepiness persisted. Similar finding was exhibited in the previous study [15] in which narcoleptic patients were diagnosed with OSA alone and it postponed the diagnosis of narcolepsy. Therefore, clinicians should be aware that many sleep-related disorders such as narcolepsy, OSA, circadian rhythm sleep-wake disorder, restless leg syndrome, insufficient sleep syndrome, and Kleine-Levin syndrome are related to EDS. Also, they should try actively to evaluate cataplexy and other symptoms of narcolepsy such as hypnagoic hallucination and sleep paralysis when a patient visits the sleep center complaining of EDS.

Chervin and Aldrich [1] described that the prevalence of EDS in OSA patients is as high as $87.2 \%$. Even in the general population with no symptoms of EDS, $6 \%$ of male and $1.5 \%$ of female report positive MSLT results (MSL $<8$ minutes and SOREMPs $\geq 2$ ). MSLT has high specificity (95\%) but falsepositive rate of $5 \%$ remains. In chronic sleep insufficient conditions such as sleep deprivation, OSA, circadian rhythm sleep disorders, depression and other medical and psychiatric disorders may show false-positive results in MSLT [16]. In the case 2-1, the patient showed a MSL 3.5 minutes and two SOREMPs. He could be diagnosed with narcolepsy without cataplexy. Meanwhile, the normal REM sleep latency is reported in the range of 50-150 mins. In narcoleptic patients, REM sleep appears within the range of 10-15 mins following the onset of sleep [17]. The case 2-1 patient's stage REM latency from sleep onset was relatively longer than that of the previous three cases.

Also, the patient's clinical symptoms started since mid-ages, after his snoring became more severe. Therefore, it is possible that his MSLT results are false-positive and we should observe further changes in the patient's clinical manifestations.

A study [15] suggests that patients who are co-diagnosed with OSA and narcolepsy should be prescribed medication such as CNS stimulants to be used prior to CPAP in treating EDS. CPAP application may initially worsen EDS in narcoleptic patients [15]. Also, another study [10] showed thirty two narcoleptic patients had an AHI $>10$, and ten of them were previously diagnosed with OSA, nine of them treated by CPAP. They showed no improvement in EDS with CPAP. Overall, medication and CPAP should be applied simultaneously in narcoleptic OSA patients. In our three cases, we applied modafinil or methylphenidate first and recommended CPAP later, which led to much improvement in ESS score and quality of daily life.

In conclusion, clinicians need to undertake intensive clinical interviews to identify sleep disorders such as narcolepsy and OSA when examining patients who are complaining of EDS. The onset and duration of EDS should be carefully examined in detail and PSG as well as MSLT recordings should be used to assist in appropriate treatment. The use of MSLT testing is essential for confirming the diagnosis of narcolepsy in patients with EDS.

\section{Conflicts of Interest}

The authors have no financial conflicts of interest.

\section{Authors' Contribution}

Conceptualization: Hong S. Data curation: Hong S. Formal analysis: Hong S. Project administration: Hong S. Resources: Hong S. Supervision: Hong S. Validation: Hong S. Writing-original draft: Kim H. Writingreview \& editing: Hong S, Song J, Kim T, Kim S, Um Y, Oh J, Cho I.

\section{REFERENCES}

1. Chervin RD, Aldrich MS. Sleep onset REM periods during multiple sleep latency tests in patients evaluated for sleep apnea. Am J Respir Crit Care Med 2000;161(2 Pt 1):426-31.

2. Seneviratne U, Puvanendran K. Excessive daytime sleepiness in obstructive sleep apnea: prevalence, severity, and predictors. Sleep Med 2004;5:339-43.

3. Aldrich MS, Chervin RD, Malow BA. Value of the multiple sleep latency test (MSLT) for the diagnosis of narcolepsy. Sleep 1997;20:620-9.

4. Young T, Palta M, Dempsey J, Skatrud J, Weber S, Badr S. The occurrence of sleep-disordered breathing among middle-aged adults. $N$ Engl J Med 1993;328:1230-5.

5. Kim MK, Lee WY, Kang JH, Kang JH, Kim BT, Kim SM, et al. 2014 clinical practice guidelines for overweight and obesity in Korea. Endocrinol Metab (Seoul) 2014;29:405-9.

6. Carpio Muñoz V, Carmona Bernal C, García Díaz E, Botebol Benhamou G, Cano Gómez S, Capote Gil F. [Association of obstructive apnea syndrome during sleep and narcolepsy]. Arch Bronconeumol 1998;34:310-1.

7. Zamarrón C, García JM, Robles A. [Obstructive sleep apnea syndrome and narcolepsy. A report of 2 cases]. Arch Bronconeumol 1998;34:466.

8. Reimão R, Lemmi H. [Concomitant narcolepsy and sleep apnea: report of a case]. Arq Neuropsiquiatr 1986;44:73-7.

9. Santamaria J, Iranzo A, Ma Montserrat J, de Pablo J. Persistent sleepiness in CPAP treated obstructive sleep apnea patients: evaluation and treatment. Sleep Med Rev 2007;11:195-207.

10. Frauscher B, Ehrmann L, Mitterling T, Gabelia D, Gschliesser V, Brandauer E, et al. Delayed diagnosis, range of severity, and multiple sleep comorbidities: a clinical and polysomnographic analysis of 100 patients of the innsbruck narcolepsy cohort. J Clin Sleep Med 2013;9: 805-12.

11. Schuld A, Hebebrand J, Geller F, Pollmächer T. Increased body-mass index in patients with narcolepsy. Lancet 2000;355:1274-5.

12. Heier MS, Jansson TS, Gautvik KM. Cerebrospinal fluid hypocretin 1 deficiency, overweight, and metabolic dysregulation in patients with narcolepsy. J Clin Sleep Med 2011;7:653-8.

13. Chabas D, Foulon C, Gonzalez J, Nasr M, Lyon-Caen O, Willer JC, et al. Eating disorder and metabolism in narcoleptic patients. Sleep 2007; 30:1267-73.

14. Chakravorty SS, Rye DB. Narcolepsy in the older adult: epidemiology, diagnosis and management. Drugs Aging 2003;20:361-76.

15. Sansa G, Iranzo A, Santamaria J. Obstructive sleep apnea in narcolepsy. Sleep Med 2010;11:93-5.

16. Aldrich MS. Narcolepsy. N Engl J Med 1990;323:389-94.

17. Mosko SS, Shampain DS, Sassin JF. Nocturnal REM latency and sleep disturbance in narcolepsy. Sleep 1984;7:115-25. 